\title{
Erratum to: Radio core dominance of Fermi blazars
}

\author{
Zhi-Yuan Pei ${ }^{1,2}$ - Jun-Hui Fan ${ }^{1,2} \cdot$ Yi Liu $^{1,2} \cdot$ Yu-Hai Yuan ${ }^{1,2}$ - Wei Cai ${ }^{1,2}$. \\ Hu-Bing Xiao ${ }^{1,2}$. Chao Lin ${ }^{1,2}$ • Jiang-He Yang ${ }^{3}$
}

Published online: 5 July 2016

(C) Springer Science+Business Media Dordrecht 2016

Erratum to: Astrophys Space Sci (2016) 361:237

DOI 10.1007/s10509-016-2822-0

There was an error in the fourth author's name in the initial online publication. The online publication has been updated to address the error, and the author's name is also correct in this erratum.

The online version of the original article can be found under doi:10.1007/s10509-016-2822-0.

$凶$ J.-H. Fan

fjh@gzhu.edu.cn

1 Center for Astrophysics, Guangzhou University, Guangzhou 510006, China

2 Astronomy Science and Technology Research Laboratory of Department of Education of Guangdong Province, Guangzhou 510006, China

3 Department of Physics and Electronics Science, Hunan University of Arts and Science, Changde 415000, China 